\title{
Caraway yellows virus, a novel nepovirus from Carum carvi
}

 \\ Jonas Hartrick', Christina Maaß ${ }^{1}$, Roswitha Ulrich ${ }^{2}$ and Heiko Ziebell ${ }^{1 *}$ (i)
}

\begin{abstract}
A novel nepovirus was identified and characterised from caraway, and tentatively named caraway yellows virus (CawYV). Tubular structures with isomeric virus particles typical for nepoviruses were observed in infected tissues by electron microscopy. The whole genome of CawYV was identified by high throughput sequencing (HTS). It consists of two segments with $8026 \mathrm{nt}$ for RNA1 and $6405 \mathrm{nt}$ for RNA2, excluding the poly(A) tails. CawYV-RNA1 shared closest nt identity to peach rosette mosaic virus (PRMV) with 63\%, while RNA2 shared $41.5 \%$ with blueberry latent spherical virus (BLSV). The amino acid sequences of the CawYV protease-polymerase (Pro-Pol) and capsid protein (CP) regions share the highest identities with those of the subgroup $C$ nepoviruses. The Pro-Pol region shared highest aa identity with PRMV (80.1\%), while the CP region shared 39.6\% to soybean latent spherical virus.

Phylogenetic analysis of the CawYV-Pro-Pol and -CP aa sequences provided additional evidence of their association with nepoviruses subgroup C. Based on particle morphology, genomic organization and phylogenetic analyses, we propose CawY as a novel species within the genus Nepovirus subgroup C.
\end{abstract}

Keywords: Caraway, High throughput sequencing, Bipartite genome, Tubular structures, Nepovirus subgroup C

\section{Main text}

Viruses from the genus Nepovirus in the subfamily Comovirinae of the Secoviridae family possess a bipartite genome consisting of two positive ssRNAs with a $5^{\prime}$ viral protein genome-linked (VPg) and a 3' poly(A) tail [1]. The RNA1 segment encodes the helicase, protease and its cofactor, replicase and the viral protein genome linked whereas the RNA2 segment encodes the movement and coat proteins $[1,2]$. Nepoviruses are the only members of the family Secoviridae known to have a single CP [2]. Each of the two RNA segments are encapsidated separately in a non-enveloped icosahedral virion of $25-30 \mathrm{~nm}$ in diameter [1]. Nepoviruses can be transmitted non-persistently and non-circulatively by nematodes, mite and thrips $[1,3]$. Seed and pollen transmissions are well-documented [1, 2]. In herbaceous plants, the symptoms induced are often transient with symptom recovery being a common outcome [2].

\footnotetext{
* Correspondence: heiko.ziebell@julius-kuehn.de

${ }^{1} J$ ulius Kühn Institute, Institute for Epidemiology and Pathogen Diagnostics, Messeweg 11-12, 38104 Braunschweig, Germany

Full list of author information is available at the end of the article
}

Caraway (Carum carvi L.) is an aromatic biennial plant in the Apiaceae family [4]. It is native to Europe, north Africa and western Asia $[5,6]$. Caraway is used as a food flavour, fragrance additive, and for medical purposes as an antibacterial agent with antispasmodic, carminative, and appetite stimulant properties [4]. In 2016, an organic caraway field showed crop losses. A caraway plant sample with systemic yellowing was sent to Julius Kuehn-Institute (JKI) for analysis (Fig. 1a). The sample tested positive by DAS-ELISA using antiserum JKI 1283 developed against an uncharacterised nepovirus from carrot which is likely a strain of cherry leaf roll virus (CLRV) (unpublished data). The virus was mechanically transmitted to Nicotiana benthamiana and chlorotic local lesions were observed on inoculated leaves followed by systemic chlorosis and necrosis. Symptom recovery was not observed. The virus was provisionally named "caraway yellows virus" (CawYV).

Electron microscopy (EM) revealed the presence of isomeric virus particles of about $30 \mathrm{~nm}$ in diameter in preparations made from the original infected caraway sample (Fig. 1b), indicating the presence of a nepovirus. Additionally, tubules containing virus-like particles in

(c) The Author(s). 2019 Open Access This article is distributed under the terms of the Creative Commons Attribution 4.0 International License (http://creativecommons.org/licenses/by/4.0/), which permits unrestricted use, distribution, and 

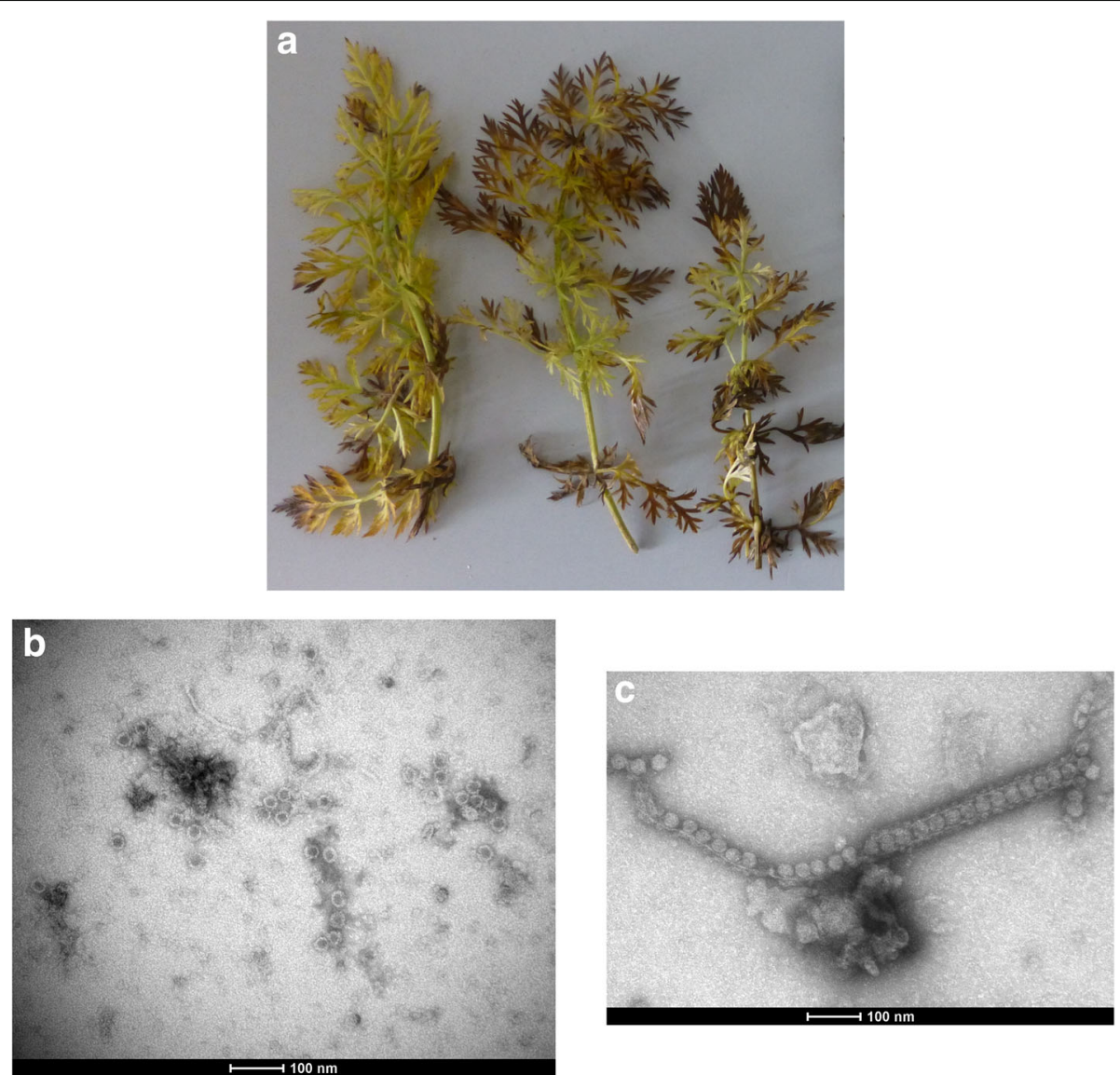

Fig. 1 (a) Leaf symptoms of caraway yellows virus (CawY) on caraway plants; (b) Electron micrograph of CawYV particles from the original infected caraway sample; (c) Electron micrograph showing tubular structure containing virus particles of CawYV in tissue homogenate of infected Nicotiana benthamiana

tissue homogenate of $N$. benthamiana infected with the nepovirus were also observed by EM (Fig. 1c). This has also been shown for other nepoviruses e.g., grapevine fanleaf virus, where the movement and the capsid proteins act as components of tubular structures (required for cell to cell movement) that traverse the cell wall with the virus particles $[1,7]$.

To obtain the full genome of CawYV, double stranded RNA (dsRNA) was extracted from infected $N$. benthamiana using Double-RNA Viral dsRNA Extraction Mini Kit for Plant Tissue (iNtRON) following the manufacturer's instructions. The extracted dsRNA was sent for library preparation and high throughput sequencing (HTS) at Eurofins GATC Biotech GmbH. The dsRNA was fragmented, strand specific cDNA was synthesized using random primers (the dsRNA was denatured at $99^{\circ}$ $\mathrm{C}$ for $2 \mathrm{~min}$ ), followed by adapter ligation and adapter specific PCR amplification then sequencing on Illumina NovaSeq 6000 platform $(2 \times 150)$.

Using Geneious Prime (v. 2019.0.4), the raw reads (15, $468,416)$ were quality trimmed, filtered, normalized, and error corrected followed by de novo assembly using
Geneious assembler (Medium sensitivity/Fast setting). 36,634 contigs of lengths between 100 and 23,141 nt were generated. A BLASTn search of the contigs against a local database for viruses and viroids downloaded from NCBI showed that two contigs of 7180 and 6341 nt had $72 \%$ identity (73\% coverage and zero E-value) to peach rosette mosaic virus (PRMV) and 79\% (16\% coverage and 1e-90 E-value) to blueberry latent spherical virus (BLSV), respectively. The 5' ends of both RNA segments were confirmed using RNA ligase-mediated amplification of cDNA ends (RLM-RACE) [8]. The 3' ends of the two RNA segments were determined by using an oligo(d)T primer for cDNA synthesis followed by PCR using virus specific primers and the oligo(d)T primer. The primers used for the $5^{\prime}$ and $3^{\prime}$ ends confirmation are listed in Additional file 1: Table S1. The PCR products were cloned, sequenced and the resulting sequences were assembled using the map to reference tool and the original assembled contigs as references. 72,977 of the quality trimmed reads were assembled to CawYV complete genome. The assembled genome of CawYV was 8026 nt for RNA segment 1 and 6405 nt for RNA 
segment 2 (excluding poly(A) tails). The sequences were deposited in the GenBank database under accessions MK492273 and MK492274. For diagnostic purposes and to confirm the presence of CawYV in symptomatic leaf tissue, a primer pair was designed using Primer 3 tool in Geneious (HZ-636 5' TGA AGA TCC GGG AAA GGC AC 3' and HZ-637 5' ACG CTT TCC ACT CTC ACC TG 3') [9]. The presence of CawYV was confirmed in the infected plants by RT-PCR using OneTaq One-Step RT-PCR Kit (NEB) resulting in amplicons of $481 \mathrm{bp}$ (data not shown).
Further analyses of the CawYV sequence confirmed its identity as a nepovirus. In analogy to other nepoviruses, CawYV RNA1 contains an open reading frame (nt position 92 to 6733) encoding a polyprotein of 2213 aa in length. Pairwise comparisons of nt and aa sequences of this ORF to its homologues of the other nepoviruses were performed using ClustalW [10]. The results show that the highest similarity was shared with PRMV at $66.1 \%$ on nt and $68.1 \%$ aa levels, respectively (Table 1 ). By searching for the different nepovirus motifs using the motif searching tool in Geneious, the locations of the

Table 1 Characteristics and pairwise nucleotide (nt) and amino acid (aa) alignments of the different regions of caraway yellows virus (CawYV) and selected members of subgroup C of the genus Nepovirus i.e., blueberry latent spherical virus (BLSV), blackcurrant reversion virus (BCRV), cherry leaf roll virus (CLRV), grapevine Bulgarian latent virus (GBLV), peach rosette mosaic virus (PRMV), soybean latent spherical virus (SLSV) and tomato ringspot virus (ToRSV)

\begin{tabular}{|c|c|c|c|c|c|c|c|c|c|c|}
\hline \multicolumn{3}{|l|}{ Virus } & \multirow{2}{*}{$\frac{\text { CawYV }}{\text { MK492273 }}$} & \multirow{2}{*}{$\begin{array}{l}\text { PRMV } \\
\text { NC_034214 }\end{array}$} & \multirow{2}{*}{$\begin{array}{l}\text { BLSV } \\
\text { NC_038764 }\end{array}$} & \multirow{2}{*}{$\begin{array}{l}\text { SLSV } \\
\text { NC_032270 }\end{array}$} & \multirow{2}{*}{$\begin{array}{l}\text { BCRV } \\
\text { NC_003509 }\end{array}$} & \multirow{2}{*}{$\begin{array}{l}\text { GBLV } \\
\text { NC_015492 }\end{array}$} & \multirow{2}{*}{$\begin{array}{l}\text { ToRSV } \\
\text { NC_003840 }\end{array}$} & \multirow{2}{*}{$\begin{array}{l}\text { CLRV } \\
\text { NC_015414 }\end{array}$} \\
\hline nt length & RNA 1 & Accession no. & & & & & & & & \\
\hline & & Complete /-poly(A) & 8026 & 8014 & 7960 & 8170 & 7711 & 7452 & 8214 & 7918 \\
\hline & & 5'UTR & 91 & 41 & 61 & 13 & 66 & 87 & 77 & 11 \\
\hline & & ORF & 6642 & 6504 & 6519 & 6588 & 6285 & 6288 & 6594 & 6339 \\
\hline & & 3'UTR & 1293 & 1469 & 1380 & 1569 & 1360 & 1077 & 1543 & 1568 \\
\hline & RNA 2 & Accession no. & MK492274 & NC_034215 & NC_038763 & NC_032271 & NC_003502 & NC_015493 & NC_003839 & NC_015415 \\
\hline & & Complete /-poly(A) & 6405 & 5956 & 6344 & 5776 & 6405 & 5821 & 7271 & 6360 \\
\hline & & $5^{\prime} U T R$ & 94 & 47 & 55 & 23 & 161 & 189 & 75 & 11 \\
\hline & & ORF & 5022 & 4425 & 4896 & 4197 & 4881 & 4500 & 5649 & 4770 \\
\hline & & $3^{\prime} \mathrm{UTR}$ & 1289 & 1484 & 1393 & 1556 & 1363 & 1132 & 1547 & 1579 \\
\hline \multicolumn{11}{|c|}{ Pairwise identity \% } \\
\hline \multirow[t]{8}{*}{ nt $\%$} & RNA 1 & Complete /-poly(A) & & 63 & 60.8 & 49.6 & 38.5 & 37 & 36 & 33.8 \\
\hline & & $5^{\prime}$ UTR & & 56.1 & 55.7 & 30.8 & 40.9 & 31.6 & 52 & 63.6 \\
\hline & & ORF & & 66.1 & 62.4 & 53.5 & 40.4 & 39.2 & 36.7 & 36.3 \\
\hline & & $3^{\prime} \mathrm{UTR}$ & & 51.4 & 53.8 & 34 & 40.5 & 36.5 & 27 & 30.9 \\
\hline & RNA 2 & Complete /-poly(A) & & 41.3 & 41.5 & 37.7 & 35.9 & 30.9 & 38.6 & 35.6 \\
\hline & & 5'UTR & & 37.2 & 30.9 & 34.8 & 35.9 & 36.2 & 41.3 & 63.6 \\
\hline & & ORF & & 39.9 & 38.5 & 39 & 37 & 31.8 & 40.9 & 37.7 \\
\hline & & $3^{\prime} U T R$ & & 51 & 55.2 & 33.4 & 36.1 & 37.1 & 29.4 & 30.6 \\
\hline \multirow[t]{12}{*}{ aa $\%$} & RNA 1 & ORF & & 68.1 & 62.5 & 48.1 & 24.5 & 22.8 & 23.1 & 22.6 \\
\hline & & $\mathrm{X}_{1}$ & & 27.9 & 29.7 & 22.9 & 12.2 & 10.6 & 10.8 & 10.8 \\
\hline & & X2 Pro-cof & & 52.9 & 53.2 & 33.9 & 25.8 & 21.4 & 15.6 & 18.5 \\
\hline & & $\mathrm{Hel}$ & & 82.5 & 75.6 & 59.3 & 28 & 24.1 & 22.6 & 23.2 \\
\hline & & $\mathrm{VPg}$ & & 75 & 57.6 & 56.2 & 6.1 & 27.3 & 25.9 & 37.9 \\
\hline & & Pro & & 79 & 66.8 & 51.3 & 27.6 & 23.5 & 24.7 & 23.1 \\
\hline & & RdRp & & 75.5 & 68.6 & 53.9 & 33.8 & 33.6 & 33.7 & 30.9 \\
\hline & & Pro-Pol & & 80.1 & 70.2 & 54.9 & 7.1 & 35.3 & 36.5 & 34.6 \\
\hline & RNA 2 & ORF & & 22.9 & 20.1 & 19 & 13.1 & 12.2 & 22.3 & 19 \\
\hline & & $\mathrm{HP}$ & & 23.3 & 13.1 & 6.5 & 7.3 & 7.6 & 8.2 & 7 \\
\hline & & $\mathrm{MP}$ & & 10 & 8.4 & 7.5 & 8.5 & 4.1 & 52.2 & 54.6 \\
\hline & & $C P$ & & 36.5 & 34.7 & 39.6 & 24.3 & 24.8 & 26.1 & 20 \\
\hline
\end{tabular}


putative protease cofactor (Pro-cof), the NTP-binding helicase domain (Hel), the serine protease domain (Pro), and the RNA-dependent RNA polymerase (RdRp) core domain were found in the RNA1-encoded polyprotein [11]. The putative viral protease cofactor motif $\left(\mathrm{FX}_{27} \mathrm{WX}_{11} \mathrm{LX}_{21} \mathrm{LXE}\right)$ was located at aa residues 438 502. The typical NTP-binding helicase motif A $\left(\mathrm{GX}_{4} \mathrm{GKS}\right)$, motif $\mathrm{B}(\mathrm{D})$, and motif $\mathrm{C}(\mathrm{N})$ were found at aa $752-759,803$, and 852 , respectively. A serine protease motif was found at aa 1280-1449 $\left(\mathrm{HX}_{40} \mathrm{EX}_{106} \mathrm{SGX}_{8} \mathrm{GX}_{5} \mathrm{GXHX}_{2} \mathrm{G}\right)$ of the CawYV RNA1 polyprotein sequence (Fig. 2a). The serine at this position is unusual for members of the Picornavirales (where cysteine is usually encoded) but was described for some members of genus Nepovirus subgroup C i.e., BLSV, CLRV, PRMV and soybean latent spherical virus (SLSV). The RNA-dependent RNA polymerase (RdRp) core domain was located at aa 1774-1880 $\left(\mathrm{DX}_{4} \mathrm{DX}_{56} \mathrm{GX}_{3} \mathrm{TX}_{3} \mathrm{NX}_{33} \mathrm{GDD}\right)$. Pairwise analysis of the protease-polymerase (Pro-Pol) region aa sequences showed a closest identity to PRMV Pro-Pol with $80.1 \%$ (Table 1).

CawYV-RNA2 contains an open reading frame (nt position 95 to 5116) encoding a polyprotein of 1673 aa in length. Pairwise comparisons of RNA2-ORF nt and aa sequences to the homologues of other nepoviruses showed the highest similarity with PRMV with $31 \% \mathrm{nt}$ and $21.3 \%$ aa identities (Table 1). The conserved movement protein motif (P) was found at aa position 962 [12]. The CP N-terminal five amino acid residues (SGLEE) together with an alternate capsid protein (CP) motif (FXFYGWS) were located at aa positions 11191122 and 1631-1637 [11, 13]. Pairwise analysis of the $\mathrm{CP}$ region showed that it shares highest aa identity to SLSV (39.6\%, Table 1).

Each of the CawYV polypeptides is predicted to be proteolytically cleaved into putative peptides by the virus-encoded protease. Sequence alignment of all
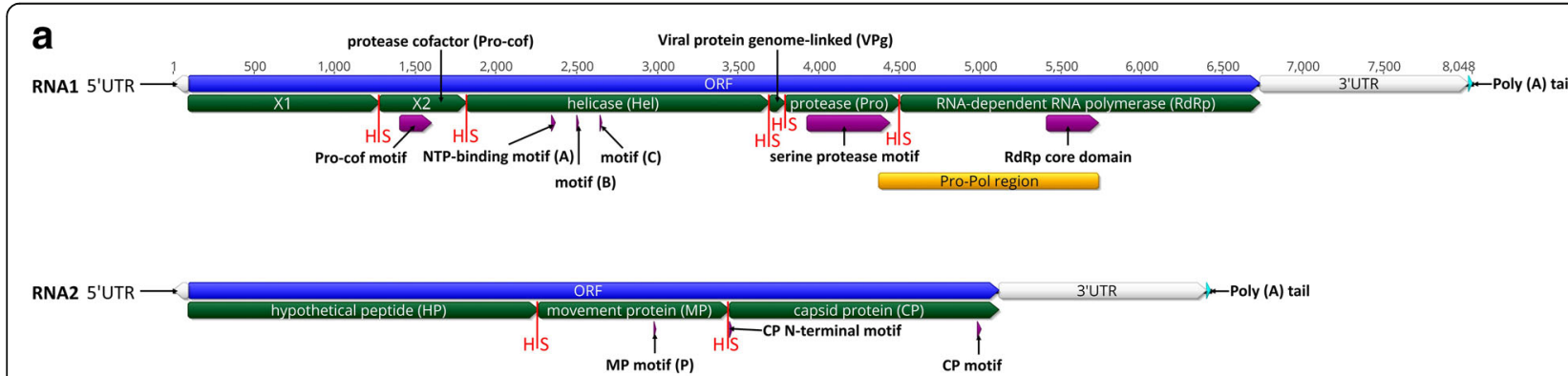

b

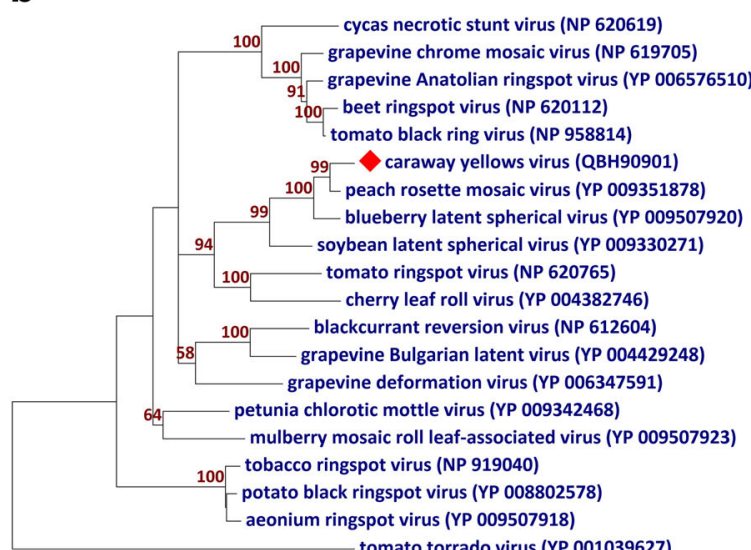

0.5
C

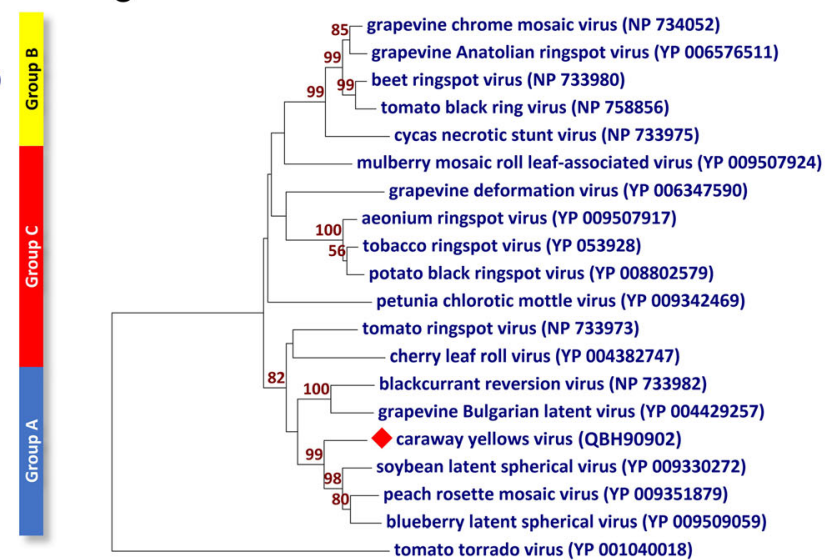

0.5

Fig. 2 (a) Genome organization of CawYV-RNA1 and -RNA2. Each of RNAs 1 and 2 contain a single large open reading frame (in blue). The predicted putative peptides are shown in green, separated by the predicted cleavage site $(H / S)(r e d)$. The RNA1 polyprotein contains X1 protein, protease cofactor (Pro-cof/X2), helicase (Hel), viral protein genome-linked (VPg), protease (Pro) and RNA-dependent RNA polymerase (RdRP). RNA2 encodes for a polyprotein with hypothetical protein (HP), movement protein (MP) and capsid protein (CP). The conserved nepovirus sequences (domains and motifs) are shown in violet. The protease-polymerase (Pro-Pol) region of CawYV-RNA1 starts with the serine (S) of the protease motif and ends with the (GDD) motif of the polymerase (shown in gold). (b) Maximum-likelihood (ML) phylogenetic trees showing the relationships between CawY and members of the genus Nepovirus based on aa alignments of the Pro-Pol region and (c) the capsid protein (CP) region. Numbers on branches indicate the bootstrap percentages (1000 replicates, $\geq 50 \%$ are shown). Tomato torrado virus (genus Torradovirus, family Secoviridae) is an outgroup 
nepovirus ORF aa sequences suggest a putative proteolytic cleavage sites at dipeptides $(\mathrm{H} / \mathrm{S})$. This potential cleavage site was not identified before in the Secoviridae members. The conserved histidine in the substratebinding pocket of the protease is known for members of the subgroup $\mathrm{C}$, however the known cleavage sites are $\mathrm{Q} / \mathrm{G}, \mathrm{Q} / \mathrm{S}$ or $\mathrm{D} / \mathrm{S}$ (confirmed experimentally) [1]. The $\mathrm{H} / \mathrm{S}$ dipeptide is also found in SLSV, BLSV and PRMV. Although the VPg motif was not confirmed in the polyprotein of RNA1, the location of the putative VPg domain could be determined by the $\mathrm{H} / \mathrm{S}$ dipeptides between the NTP-binding helicase and the protease using sequence alignment (Fig. 2a). Additionally, the X1 putative protein was identified at the $\mathrm{N}$ terminus of RNA1 polyprotein by the presence of a $\mathrm{H} / \mathrm{S}$ dipeptide potential cleavage site before the protease cofactor motif of X2 (Fig. 2a). The $5^{\prime}$ untranslated regions (UTR) of the two RNAs were $91 \mathrm{nt}$ for RNA1 and $94 \mathrm{nt}$ for RNA2 and shared $61.3 \%$ nt identity to each other. The 3'UTRs (1293 and 1289 nt for RNA 1 and 2 respectively, excluding the poly(A) tail) are $98.4 \%$ identical.

A maximum likelihood tree using MEGA7 software ( $\mathrm{v}$ 7.0.26) based on the aa alignments of the Pro-Pol and the CP regions were additional evidence for the relatedness of CawYV to the Nepovirus subgroup C (Fig. 2b and c) [14].

The International Committee on Taxonomy of Viruses (ICTV) suggests the following criteria for species demarcation [1]: distinct host range; distinct vector specificity; absence of cross-protection; differences in antigenic reactions; absence of reassortment between RNA1 and RNA2; Pro-Pol region aa $<80 \%$ and $\mathrm{CP}$ region aa $<75 \%$ identities. Although the host range was not studied, the closest relatives of CawYV, i.e., PRMV and BLSV, are not known to infect members of the Apiaceae family. The serological crossreactivity is well known for members of the same genus in the family Secoviridae [1]. This might explain why our antiserum raised against an uncharacterised strain of CLRV reacted with CawYV. Further investigations are necessary to test the antiserum against other nepoviruses, and attempts are currently underway to develop a CawYV-specific antiserum. When compared to the closest relative PRMV, the Pro-Pol region of CawYV is slightly above the species demarcation limit by $0.1 \%$. However, this was also observed for other nepoviruses e.g., beet ringspot virus (BRSV) and tomato black ring virus (TBRV) that share $89 \%$ aa identity at the Pro-Pol [1] but are yet classified as distinct species. However, the caraway virus-CP region is very different to other nepoviruses sharing only $39.6 \%$ aa identity with SLSV. Based on these results, we propose the assignment of CawYV as a new virus species within the subgroup $C$ of the genus Nepovirus. Further studies are needed to investigate the natural mode of transmission and the biological characteristics of CawYV.

\section{Additional file}

Additional file 1: Table S1. List of the primers used for caraway yellows virus $5^{\prime}$ and $3^{\prime}$ ends confirmation. (DOCX 18 kb)

\section{Abbreviations}

aa: amino acid; BCRV: blackcurrant reversion virus; BLSV: blueberry latent spherical virus; BRSV: beet ringspot virus; CawYV: caraway yellows virus; CLRV: cherry leaf roll virus; CP: capsid protein; DAS-ELISA: double antibody sandwich enzyme-linked immunosorbent assay; dsRNA: double stranded RNA; EM: electron microscopy; GBLV: grapevine Bulgarian latent virus; Hel: helicase; HTS: high throughput sequencing; ICTV: International Committee on Taxonomy of Viruses; JKI: Julius Kuehn-Institute; kDa: kilo Dalton; nm: nanometer; nt: nucleotide; PRMV: peach rosette mosaic virus; Pro: protease; Pro-cof: protease cofactor; Pro-Pol: protease-polymerase; RdRp: RNA-dependent RNA polymerase; RLM-RACE: RNA ligase-mediated amplification of CDNA ends; RT-PCR: reverse transcription polymerase chain reaction; SLSV: soybean latent spherical virus; TBRV: tomato black ring virus; ToRSV: tomato ringspot virus; UTR: untranslated regions; VPg: protein genome-linked

\section{Acknowledgments}

The authors are grateful to the reviewers for their suggestions.

\section{Authors' contributions}

YZAG and HZ conceived and designed the study; YZAG carried out molecular studies; KR-P performed the electron microscopy experiments; YZAG analysed the data; YZAG drafted the manuscript; AS, PL, KH, JH and $\mathrm{CM}$ provided technical assistance; RU provided the original sample; Funding was acquired by $\mathrm{HZ}$; All authors read and approved the final version.

\section{Funding}

This research was financed by the EUPHRESCO project (2015-F-172; The application of Next-Generation Sequencing technology for the detection and diagnosis of non-culturable organism: Viruses and viroids). Yahya Z. A. Gaafar was funded by the German Egyptian research long term scholarship. Open access publication was enabled by JKI core funding.

\section{Availability of data and materials}

The datasets used and/or analysed during the current study are available from the corresponding author on reasonable request.

\section{Ethics approval and consent to participate}

This research does not contain any studies on human or animals.

Consent for publication

All authors agreed to the publication of this manuscript.

\section{Competing interests}

The authors declare no competing interests.

\section{Author details}

${ }^{1}$ Julius Kühn Institute, Institute for Epidemiology and Pathogen Diagnostics, Messeweg 11-12, 38104 Braunschweig, Germany. ${ }^{2}$ Regierungspräsidium Gießen, Schanzenfeldstrasse 8, 325578 Wetzlar, Germany.

Received: 26 March 2019 Accepted: 16 May 2019

Published online: 27 May 2019

\section{References}

1. Thompson JR, Dasgupta I, Fuchs M, Iwanami T, Karasev AV, Petrzik K, et al. ICTV virus taxonomy profile: Secoviridae. J Gen Virol. 2017;98:529-31. https:// doi.org/10.1099/jgv.0.000779. 
2. Fuchs M, Schmitt-Keichinger C, Sanfaçon H. Chapter two - a renaissance in nepovirus research provides new insights into their molecular interface with hosts and vectors. In: Kielian M, Thomas CM, Marilyn JR, editors. Adv Virus Res. Saint Louis: Elsevier Science; 2017. p. 61-105. https://doi.org/10.1016/bs. aivir.2016.08.009.

3. Bergeson GB, Athow KL, Laviolette FA, Thomasine M. Transmission, movement, and vector relationships of tobacco ringspot virus in soybean. Phytopathology. 1964:54:723-8.

4. lacobellis NS, Lo Cantore P, Capasso F, Senatore F. Antibacterial activity of Cuminum cyminum L. and Carum carvi L. essential oils. J Agric Food Chem. 2005;53:57-61. https://doi.org/10.1021/jf0487351.

5. Bailer J, Aichinger T, Hackl G, de HK, Dachler M. Essential oil content and composition in commercially available dill cultivars in comparison to caraway. Ind Crop Prod. 2001;14:229-39. https://doi.org/10.1016/S09266690(01)00088-7

6. Eddouks M, Lemhadri A, Michel J-B. Caraway and caper: potential antihyperglycaemic plants in diabetic rats. J Ethnopharmacol. 2004;94:143-8. https://doi.org/10.1016/j.jep.2004.05.006 .

7. Laporte C, Vetter G, Loudes A-M, Robinson DG, Hillmer S, Stussi-Garaud C, Ritzenthaler C. Involvement of the secretory pathway and the cytoskeleton in intracellular targeting and tubule assembly of Grapevine fanleaf virus movement protein in tobacco BY-2 cells. Plant Cell. 2003;15:2058-75. https://doi.org/10.1105/tpc.013896.

8. Coutts RHA, Livieratos IC. A rapid method for sequencing the 5'- and 3'-termini of double-stranded RNA viral templates using RLM-RACE.J Phytopathol. 2003; 151:525-7. https://doi.org/10.1046/.1439-0434.2003.00755.x .

9. Untergasser A, Cutcutache I, Koressaar T, Ye J, Faircloth BC, Remm M, Rozen SG. Primer3--new capabilities and interfaces. Nucleic Acids Res. 2012;40: e115. https://doi.org/10.1093/nar/gks596

10. Larkin MA, Blackshields G, Brown NP, Chenna R, McGettigan PA, McWilliam $H$, et al. Clustal W and Clustal X version 2.0. Bioinformatics. 2007;23:2947-8. https://doi.org/10.1093/bioinformatics/btm404 .

11. Isogai M, Tatuto N, Ujiie C, Watanabe M, Yoshikawa N. Identification and characterization of blueberry latent spherical virus, a new member of subgroup C in the genus Nepovirus. Arch Virol. 2012;157:297-303. https:// doi.org/10.1007/s00705-011-1177-7.

12. Mushegian AR. The putative movement domain encoded by nepovirus RNA-2 is conserved in all sequenced nepoviruses. Arch Virol. 1994;135:43741. https://doi.org/10.1007/BF01310028

13. Le Gall O, Candresse T, Dunez J. A multiple alignment of the capsid protein sequences of nepoviruses and comoviruses suggests a common structure. Arch Virol. 1995;140:2041-53. https://doi.org/10.1007/BF01322691

14. Kumar S, Stecher G, Tamura K. MEGA7: molecular evolutionary genetics analysis version 7.0 for bigger datasets. Mol Biol Evol. 2016;33:1870-4. https://doi.org/10.1093/molbev/msw054

\section{Publisher's Note}

Springer Nature remains neutral with regard to jurisdictional claims in published maps and institutional affiliations.

Ready to submit your research? Choose BMC and benefit from:

- fast, convenient online submission

- thorough peer review by experienced researchers in your field

- rapid publication on acceptance

- support for research data, including large and complex data types

- gold Open Access which fosters wider collaboration and increased citations

- maximum visibility for your research: over $100 \mathrm{M}$ website views per year

At $\mathrm{BMC}$, research is always in progress.

Learn more biomedcentral.com/submissions 